\title{
Chronic neural recordings using silicon microelectrode arrays electrochemically deposited with a poly (3,4-ethylenedioxythiophene) (PEDOT) film*
}

\author{
Kip A Ludwig ${ }^{1}$, Jeffrey D Uram ${ }^{1}$, Junyan Yang ${ }^{2}$, David C Martin ${ }^{1,4,5}$ \\ and Daryl R Kipke ${ }^{1,3}$ \\ ${ }^{1}$ Department of Biomedical Engineering, University of Michigan, Ann Arbor, MI 48109, USA \\ ${ }^{2}$ Dow Chemical Co., Freeport, TX 77541, USA \\ ${ }^{3}$ Department of Electrical Engineering and Computer Science, University of Michigan, Ann Arbor, \\ MI 48109, USA \\ ${ }^{4}$ Materials Science and Engineering, University of Michigan, Ann Arbor, MI 48109, USA \\ ${ }^{5}$ Macromolecular Science and Engineering Center, University of Michigan, Ann Arbor, MI 48109, USA \\ E-mail: ludwigk@umich.edu and dkipke@engin.umich.edu
}

Received 21 November 2005

Accepted for publication 8 February 2006

Published 1 March 2006

Online at stacks.iop.org/JNE/3/59

\begin{abstract}
Conductive polymer coatings can be used to modify traditional electrode recording sites with the intent of improving the long-term performance of cortical microelectrodes. Conductive polymers can drastically decrease recording site impedance, which in turn is hypothesized to reduce thermal noise and signal loss through shunt pathways. Moreover, conductive polymers can be seeded with agents aimed at promoting neural growth toward the recording sites or minimizing the inherent immune response. The end goal of these efforts is to generate an ideal long-term interface between the recording electrode and surrounding tissue. The goal of this study was to refine a method to electrochemically deposit surfactant-templated ordered poly(3,4-ethylenedioxythiophene) (PEDOT) films on the recording sites of standard 'Michigan' probes and to evaluate the efficacy of these modified sites in recording chronic neural activity. PEDOT-coated site performance was compared to control sites over a six-week evaluation period in terms of impedance spectroscopy, signal-to-noise ratio, number of viable unit potentials recorded and local field potential recordings. PEDOT sites were found to outperform control sites with respect to signal-to-noise ratio and number of viable unit potentials. The benefit of reduced initial impedance, however, was mitigated by the impedance contribution of typical silicon electrode encapsulation. Coating sites with PEDOT also reduced the amount of low-frequency drift evident in local field potential recordings. These findings indicate that electrode sites electrochemically deposited with PEDOT films are suitable for recording neural activity in vivo for extended periods. This study also provided a unique opportunity to monitor how neural recording characteristics develop over the six weeks following implantation.
\end{abstract}

\footnotetext{
* This work was supported by the Center for Wireless Integrated Microsystems NSF EEC-9986866 and the Whitaker Foundation.
}

\section{Introduction}

Implantable electrodes designed to record action potentials from individual neurons are integral to a number of clinical 
and research applications [1-6]. The ability to record action potentials from individual neurons is dependent on a tradeoff between the geometric area of the recording site and the site's impedance, often referred to as the tradeoff between selectivity and sensitivity [7, 8]. A recording site with small geometric area is required to isolate the action potential of an individual neuron from more distant neural sources (selectivity) $^{6}$ [7-11]. A low-impedance recording site is necessary because impedance is proportional to both thermal noise and signal loss through shunt pathways (sensitivity) [7-9, 11, 12]. Unfortunately, decreasing the geometric area of a recording site causes an increase in the impedance of the recording site [7-9].

A number of studies have explored methods to reduce the impedance of small recording sites in order to alleviate this tradeoff [7-9]. Fabricating non-planar structures onto the surface of the recording site, iridium oxide deposition and platinum black deposition have all been used to increase the surface area of a recording site by increasing its fractal dimensions [7-9, 12-14]. However, there are drawbacks to each of these methods. The fabrication of non-planar structures on the surface of a recording site is limited by photolithographic resolution and non-conformal metal deposition. Recording sites deposited with iridium oxide and platinum black have been reported to have unstable impedance, weak adherence to the underlying substrate and can cause drift in the open circuit potential of the site [7-9, 14]. Moreover, there has been no detailed investigation into how effective these methods are in improving the chronic performance of neural recording electrodes.

More recently, investigators have focused on the use of conductive polymers such as polypyrrole (PPy) to reduce the impedance of recording sites. The specificity attainable when electrochemically depositing conductive polymers facilitates the generation of conductive polymer films that maximize surface area over a given geometric space [15-18]. As a result, the capacitance of the recording site dramatically increases, creating a corresponding reduction in site impedance [15-17, 19]. In addition, porous conductive polymer films promote effective ion exchange between the recording site and the surrounding tissue $[15-17,19]$. These films transduce ionic current into electronic current via an efficient redox reaction, minimizing the charge transfer resistance at the recording site, again lowering the impedance of the recording site [15-17, 19].

PPy can also be used to facilitate a number of innovative techniques intended to improve neural recordings. Studies have indicated that topographically modified surfaces are capable of enhancing axonal and dendritic growth [20-23]. Electrical stimulation through oxidized PPy has also been

6 The extracellular potential field of a neuron drops off steeply with respect to distance from the neuron. Large recording sites span multiple diminishing potential lines, and therefore average strong signal from near the neuron, with weaker, more distal signal. Moreover, large recording sites are more likely to be closer to additional neurons than small recording sites, and therefore measure unwanted competing signals. Finally, recording sites that are larger than the neurons they record from run the risk of recording from both poles of a dipole source at once, effectively shorting the measured potential. demonstrated to significantly increase the neurite lengths in rat PC-12 cells in vitro [24]. Furthermore, PPy can be electrochemically deposited on microelectrode arrays and seeded with various biomolecule combinations that improve the interface between tissue and recording site [15, 16, 18]. These biomolecules can be selected to induce the attachment of cells onto coated recording sites or alleviate the inherent immune response.

Unfortunately, PPy is not well suited for chronic, longterm implantation [25-27]. A study by Yamato et al found that PPy/poly(styrene sulfonate) (PSS) retained only 5\% of its original charge after polarization at $0.4 \mathrm{~V}$ for $16 \mathrm{~h} \mathrm{[26].} \mathrm{A}$ number of studies have suggested that a nucleophilic attack of $\mathrm{OH}^{-}$on the $\alpha$ and $\beta$ positions of the pyrrole rings may lead to a loss of conjugation, and consequently a loss of electrochemical activity $[25,27,28]$.

Past studies have indicated that nodular poly(3,4ethylenedioxythiophene) (PEDOT) can be used as a viable alternative to PPy for chronic, long-term implantations $[15,17,19,26]$. Unlike PPy, PEDOT has a dioxyethylene bridging group across the 3- and 4-positions of the heterocycle preventing $\alpha-\beta^{\prime}$ coupling, and as a result is more electrochemically stable than PPy [15, 19, 26]. Yamato et al reported that PEDOT/PSS retained $89 \%$ of its original electrochemical activity when polarized at $0.4 \mathrm{~V}$ for $16 \mathrm{~h}$ [26]. PEDOT has also been effectively used as a template to incorporate the bioactive peptide DCDPGYIGSR [15]. Preferential growth of neuronal cells was observed on the coated areas of standard acute 'Michigan' probes coated with PEDOT/DCDPGYIGSR in vitro [15]. PEDOT/DCDPGYISR-coated sites were then used to obtain high-quality acute neural recordings [15].

Surfactant-templated ordered PEDOT films have been shown to possess superior electrical characteristics to the more conventional nodular PEDOT [17]. SEM micrographs indicate that surfactant-templated ordered PEDOT films have a larger surface area than nodular PEDOT or PPy films when grown under the same conditions [17]. As a result, surfactant-templated ordered PEDOT films lower recording site impedance even further than nodular PEDOT or PPy films [17]. Surfactant-templated ordered PEDOT films also demonstrate a larger charge carrying capacity than nodular PEDOT or PPy films [17]. In addition, the charge carrying capacity of surfactant-templated ordered PEDOT films has been found to be more stable than nodular PEDOT or PPy films after 1000 cyclic voltammetry cycles [17].

The goal of the current study was to refine a method to electrochemically deposit surfactant-templated ordered PEDOT films on the recording sites of standard Michigan chronic probes and to evaluate the efficacy of these modified sites in recording chronic neural activity [17]. Eight male Sprague-Dawley rats were implanted in motor cortex with standard $4 \times 4$, 16-channel probes and neural recordings were monitored over a six-week period. The performance of PEDOT-coated sites was compared to control sites in terms of impedance spectroscopy, signal-to-noise ratio, number of viable unit potentials recorded and local field potential recordings. This experiment also provided a 
unique opportunity to systematically investigate how neural recording characteristics develop over the six weeks following implantation.

\section{Methods}

\subsection{Silicon probes}

The micromachined silicon probes used in this experiment were provided by the University of Michigan Center for Neural Communications Technology. Design and fabrication of the probes have been described in detail elsewhere [10, 29]. Sixteen-channel chronic probes with $703 \mu \mathrm{m}^{2}$ iridium recording sites were selected for use throughout this work as these probes are routinely used to record single-unit action potentials in motor cortex.

\subsection{Electrochemical deposition and initial evaluation}

Electrochemical deposition of PEDOT in this study was accomplished using an electrochemical potentiostat/ galvanostat (Autolab PGSTAT12, Eco Chemie, Urtecht, The Netherlands) with associated General Purpose Electrochemical System (GPES) software. PEDOT doped with tetraethylammonium perchlorate and dissolved in $20 \mathrm{wt} \%$ surfactant poly(oxythylene) $)_{10}$-oleyl ether was galvanostatically deposited onto the iridium sites of the neural probes [17]. Test probes were first deposited with PEDOT in order to determine the optimum deposition characteristics for use in the chronic study. The deposition charge used on the test probes was varied from 50 to $1000 \mathrm{mC} \mathrm{cm}^{-2}$ in order to generate a wide spectrum of coating morphologies and thicknesses [17]. Coated sites were then visually inspected under a microscope and impedance spectroscopy measurements for each site were made (section 2.6). Next, each of the test probes was inserted and removed from a gelatin model of rat cortex [30]. After the insertion test, the test probes were again visually inspected under a microscope and impedance spectroscopy measurements for each site were retaken (figure 1). Only PEDOT films that were grown using the largest two deposition charges, corresponding to a coating thickness of approximately $6 \mu \mathrm{m}$ [17], detached partially from the electrode site upon insertion. Smaller film thicknesses appeared unchanged after insertion.

As a result of these preliminary tests, PEDOT films generated with a deposition charge of $260 \mathrm{mC} \mathrm{cm}^{-2}$ were chosen for in vivo testing. Films created with a deposition charge of $260 \mathrm{mC} \mathrm{cm}^{-2}$ were found to have an impedance drop comparable to the largest applied deposition charges, as well as a porous morphology conducive to ion transfer. Moreover, PEDOT films generated using this deposition charge extend only $1 \mu \mathrm{m}$ from the recording site [17] and therefore should mitigate the risk of delamination upon insertion, minimize the tissue displaced during insertion and present less of a focal point for the concentration of mechanical stress on the electrode shank. For in vivo testing, eight sites on each probe testing were deposited with surfactant-templated ordered PEDOT film. The deposited sites were staggered in relative location to prevent bias due to specific shank location

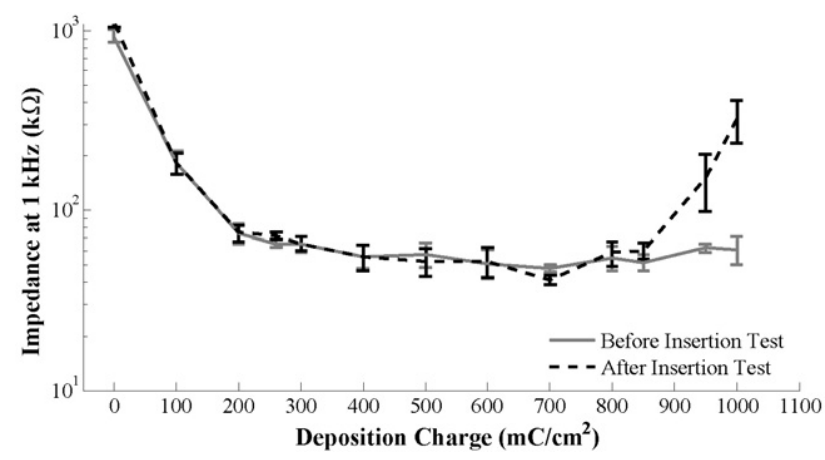

Figure 1. Average recording site impedance versus polymerization charge. The bars denote standard error $(n=4)$. Impedance at $1 \mathrm{kHz}$ decreased up to a deposition charge of $260 \mathrm{mC} \mathrm{cm}^{-2}$ and then remained relatively constant. Coatings made using the two largest deposition charges often delaminated during the insertion test, causing an increase in impedance post-insertion.

(a)

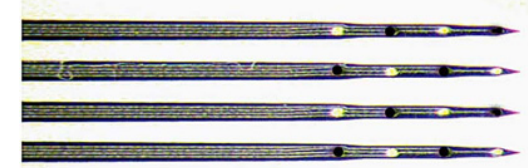

(b)

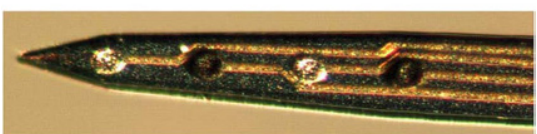

Figure 2. PEDOT coating protocol. (a) Snapshot of standard 16-site, 4-shank probe prior to deposition. The locations of electrode sites deposited with PEDOT have been artificially darkened for reference. (b) One shank of a probe after deposition. The darker sites have been electrochemically deposited with a PEDOT film. (This figure is in colour only in the electronic version)

or cortical depth (figure 2). The remaining eight sites on each probe were left uncoated as controls for comparison.

\subsection{Surgical techniques}

Eight male Sprague-Dawley rats were implanted in motor cortex with a 16-channel, chronic Michigan silicon microelectrode array using experimental procedures outlined previously [31]. Initial anesthesia was administered via intraperitoneal injections of a mixture of $50 \mathrm{mg} \mathrm{ml}^{-1}$ ketamine, $5 \mathrm{mg} \mathrm{ml}^{-1}$ xylazine and $1 \mathrm{mg} \mathrm{ml}^{-1}$ acepromazine at an injection volume of $0.125 \mathrm{ml} / 100 \mathrm{~g}$ body weight. Updates of $0.1 \mathrm{ml}$ ketamine $\left(50 \mathrm{mg} \mathrm{ml}^{-1}\right)$ were delivered as needed during the surgery to maintain anesthesia during the surgery. Animals were secured to a standard stereotaxic frame, and three stainless steel bone screws were inserted into the skull. The electrode connector was grounded to a bone screw over parietal cortex using a stainless steel ground wire. A craniotomy approximately $3 \mathrm{~mm} \times 2 \mathrm{~mm}$ craniotomy was made over motor cortex. Two incisions were made in the dura mater over the target area to create four flaps, and 
these flaps were subsequently folded back over the edge of the craniotomy. The electrodes were then hand inserted into the approximate target cortical area (target location $3.0 \mathrm{~mm}$ anterior to bregma, $2.5 \mathrm{~mm}$ lateral from bregma and $1.4 \mathrm{~mm}$ deep from the surface of the brain) [31]. Cortical depth was estimated using the known location of the electrode sites on the individual shanks in conjunction with the known length of the individual shanks. Next, NeuroSeal ${ }^{\circledR}$ (NeuroNexus Technologies, Inc., Ann Arbor, MI) was applied as a dural sealant, the silicon cable connector was wrapped with GelFoam ${ }^{\circledR}$ (Henry Schein, Inc., Miami, FL) for protection and the entire assembly excluding the connector was enclosed using dental acrylic (Co-Oral-Ite, Dental Mfg. Co., Santa Monica, CA). Finally, sutures were used to close the skin around the acrylic and triple-antibiotic ointment was applied. All procedures complied with the United States Department of Agriculture guidelines for the care and use of laboratory animals and were approved by the University of Michigan Animal Care and Use Committee.

\subsection{Data collection}

After implantation, neural recordings and impedance spectroscopy for each animal were taken daily for the first two weeks and every other day thereafter for the remainder of the six weeks. Animals were either awake or kept lightly sedated throughout the data collection sessions. No obvious, discernible difference in neuronal activity was noted between lightly sedated and awake animals. Local field potential recordings were taken only at the end of the study.

\subsection{Neural recordings and data analysis}

Recorded neural signals were acquired using a Multi-Channel Neural Acquisition Processor (MNAP; Plexon Inc., Dallas, TX). Neural electrophysiological data for all 16 recording channels were amplified and bandpass filtered; single- and multi-unit recordings were sampled at $40 \mathrm{kHz}$ and bandpass filtered from 450 to $5000 \mathrm{~Hz}$, while local field potentials were sampled at $1 \mathrm{kHz}$ and bandpass filtered from 3 to $90 \mathrm{~Hz}$. During recording sessions, animals were placed in an electrically shielded recording booth and multiple $30 \mathrm{~s}$ segments of continuous neural recordings were taken.

Neural recording segments were analyzed offline using custom-automated MatLab (Mathworks Inc., MA) software. Candidate action potentials were discriminated from background noise based on the probability distribution of the samples in a $30 \mathrm{~s}$ segment. An amplitude threshold window was set 3.5 standard deviations above and below the mean of the sample distribution. For each peak exceeding the threshold window, a $2.4 \mathrm{~ms}$ candidate waveform snippet centered on the absolute minimum of the waveform was removed from the recorded segment and stored. The amplitude of the noise voltage for every recording site in each recorded segment was calculated after all candidate waveforms had been removed.

The store of candidate waveform snippets for a given channel was used to derive a set of orthogonal basis vectors ordered based on ability to represent the largest variation in the data set, a technique commonly known as principal component analysis [32-35]. The first three principal components were used to generate the axes of a three-dimensional component space. Each waveform in the store was transformed into a point in this three-dimensional space based on its score for each of the component axes. Individual points were grouped into clusters using fuzzy C-means clustering [36, 37]. When compared to hard clustering, fuzzy clustering reduces classification errors resulting from the synchronous firing of multiple neurons [38]. In order to determine the optimum number of clusters, the number of clusters was iteratively increased until the value for the objective function calculated for $k+1$ number of clusters was at least $55 \%$ of the value for the objective function calculated for $k$ number of clusters [39].

After clustering, waveforms with a cluster membership index of greater than 0.8 were used to determine a mean waveform for a cluster. Contributions of white noise and waveforms created by the simultaneous firing of multiple neurons generally do not have a membership index of greater than 0.8 for a particular cluster and therefore were limited using this procedure [38]. An interspike interval histogram for each cluster was generated and visually inspected for an obvious absolute refractory period as an additional measure of noise rejection. Signal amplitude for a cluster was defined as the peak-to-peak amplitude of the mean waveform for each cluster. The signal-to-noise ratio (SNR) for a given cluster was defined as follows:

$\mathrm{SNR}=$ signal amplitude $/$

$(2 \times$ calculated RMS noise voltage for recording site).

Clusters were then separated into one of four categories based on calculated SNR. Clusters with an SNR of greater than 4 were categorized as quality units. Clusters with an SNR between 3 and 4 were categorized as moderate units. Clusters with an SNR between 2 and 3 were categorized as poor units, while clusters with an SNR of less than 2 were not considered units. These four categories correspond well with observations of unit quality based on signal-to-noise ratio made in similar recording studies [40, 41].

As noted elsewhere, isolating action potentials from a specific neuron using only a single recording site as a reference is prone to classification errors [35, 42]. Although classification errors are inevitable, the number of neurons detected using the methodology presented here should accurately parallel the true underlying number of neural sources. This methodology also compared favorably with conservative manual clustering performed by experienced researchers on the same data sets, but with the advantage of being both objective and automated.

\subsection{Impedance spectroscopy measurements}

Impedance spectroscopy measurements were made using an Autolab potentiostat PGSTAT12 (Eco Chemie, Utrecht, The Netherlands) with associated frequency response analyzer (Brinkmann, Westbury, NY). Impedance measurements were made by applying a $25 \mathrm{mV}$ RMS sine wave with frequencies varied logarithmically from $10 \mathrm{~Hz}$ to $10 \mathrm{kHz}$ [43]. Prior to implantation, measurements were made by immersing the 
Table 1. Summary of results across days.

\begin{tabular}{|c|c|c|c|c|c|c|c|c|}
\hline & \multicolumn{2}{|c|}{ Average impedance $(\mathrm{M} \Omega)$} & \multicolumn{2}{|c|}{ RMS noise $(\mu \mathrm{V})$} & \multicolumn{2}{|c|}{ Signal amplitude $(\mu \mathrm{V})$} & \multicolumn{2}{|c|}{ SNR } \\
\hline & PEDOT & Control & PEDOT & Control & PEDOT & Control & PEDOT & Control \\
\hline Day 0-2 & $0.13 \pm 0.06$ & $0.98 \pm 0.08$ & $5.3 \pm 0.3$ & $8.0 \pm 0.4$ & $54.1 \pm 20$ & $65.6 \pm 22$ & $5.1 \pm 1.2$ & $4.1 \pm 1.1$ \\
\hline Day 3-15 & $1.1 \pm 0.8$ & $1.7 \pm 0.9$ & $11.4 \pm 0.5$ & $11.4 \pm 0.5$ & $112 \pm 15$ & $115 \pm 17$ & $4.8 \pm 1.8$ & $4.9 \pm 1.7$ \\
\hline Day $16-42$ & $0.81 \pm 0.3$ & $1.5 \pm 0.3$ & $12.7 \pm 0.8$ & $13.1 \pm 0.9$ & $129 \pm 15$ & $113 \pm 13$ & $5.1 \pm 1.2$ & $4.3 \pm 1.0$ \\
\hline
\end{tabular}

electrode recording sites in $0.1 \mathrm{M}$ phosphate buffer saline (PBS) and a platinum foil was used as the reference electrode. After implantation, a distant stainless steel (316-SS grade) bone screw was used as the reference electrode.

\subsection{Statistical analysis}

For this study, comparative statistical significance between groups was determined using standard analysis of variance techniques (ANOVA). There were 64 PEDOT modified sites and 64 control sites on any specific day in the experiment. The factors used in initial comparative ANOVA calculations for any given metric were coated versus control and day number. A significant difference across days was noted $(p<0.001)$, but no post hoc test to separate specific days was performed, as there was an obvious day-to-day trend in the data. Instead, the data sets were grouped into three time segments (day 0-2 after surgery, day 3-15 after surgery and day 16-42 after surgery), and a one factor ANOVA (coated versus control) was performed. These three periods of time were chosen based on the time course of initial trauma, early reactive response and sustained immune response evident in other experiments [31, 44-46]. The associated calculated standard deviation has been included in the text with all average measurements.

\section{Results}

\subsection{Impedance spectroscopy measurements}

The impedance of electrode recording sites at $1 \mathrm{kHz}$ is often used to evaluate recording probes because action potentials have a characteristic frequency band centered at $1 \mathrm{kHz}$ [47]. Three distinct periods of time are evident in the chart of recording site impedance at $1 \mathrm{kHz}$ over time figure (3). Over the course of the first three days following surgery, the average $1 \mathrm{kHz}$ impedance for the electrode recording sites remained relatively stable. During this first period, the mean $1 \mathrm{kHz}$ impedance for the uncoated sites was $0.98 \mathrm{M} \Omega \pm 0.08 \mathrm{M} \Omega$ while the mean $1 \mathrm{kHz}$ impedance for the PEDOT recording sites was $0.13 \mathrm{M} \Omega \pm 0.06 \mathrm{M} \Omega$, a ratio of over 7 to $1(p<$ $0.001)$. The variability in $1 \mathrm{kHz}$ impedance from PEDOT site to PEDOT site and control site to control site was small. The second period of time, spanning from day 3 to day 15, was notable for an increase in both impedance magnitude and siteto-site variability. Across this second time segment, the mean $1 \mathrm{kHz}$ impedance for the control sites was $1.7 \mathrm{M} \Omega \pm 0.9 \mathrm{M} \Omega$ while the mean $1 \mathrm{kHz}$ impedance for the PEDOT sites was $1.1 \mathrm{M} \Omega \pm 0.8 \mathrm{M} \Omega$, a ratio of approximately 1.5 to $1(p<$ $0.001)$. The third period of time, spanning from day 15 to the

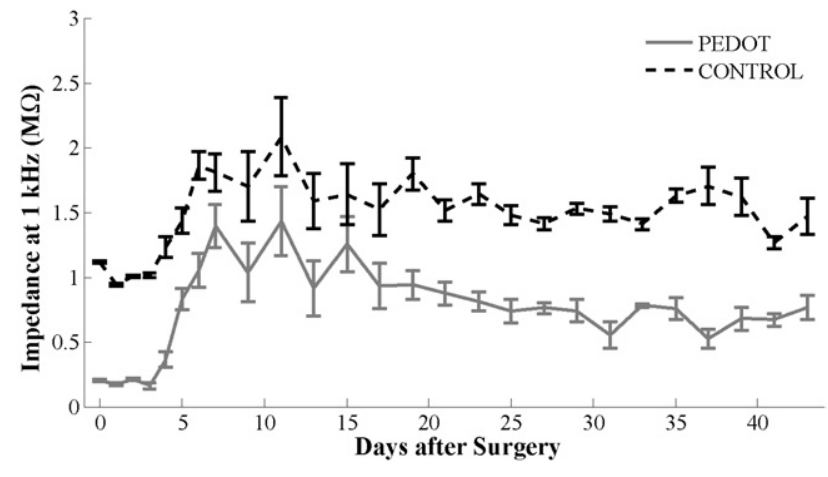

Figure 3. Average site impedances at $1 \mathrm{kHz}$ over time. The bars denote standard error of the data set on a given day $(n=64)$. Day 0 measurements were taken immediately after surgery. The $1 \mathrm{kHz}$ impedance for both PEDOT and control sites increased an average of $70 \mathrm{k} \Omega$ immediately upon implantation. Impedances increased dramatically the third day after implantation, up to a maximum value at the one-week mark. Two weeks after implantation, the variability of $1 \mathrm{kHz}$ impedance for PEDOT and control sites decreased. This trend in $1 \mathrm{kHz}$ impedance is highly correlated between PEDOT and control sites, suggesting a global immune response equally affecting both PEDOT and control sites.

end of the experiment, was marked by a reduction in day-today and site-to-site impedance variability (see table 1). During this third segment of time, the mean $1 \mathrm{kHz}$ impedance for control recording sites was $1.5 \mathrm{M} \Omega \pm 0.3 \mathrm{M} \Omega$ while the mean 1 $\mathrm{kHz}$ impedance for the PEDOT sites was $0.81 \mathrm{M} \Omega \pm 0.3 \mathrm{M} \Omega$, a ratio of roughly 2 to $1(p<0.001)$. The three distinct segments of time noted in the data set correspond well to the time course of initial trauma, early reactive response and sustained immune response evident in other experiments studying the immune response of cortical tissue to an implanted silicon probe [31, 44-46]. An increase in impedance at $1 \mathrm{kHz}$ over the two weeks following implantation was also noted in another study of Michigan probe performance conducted at the University of Michigan [31].

Bode plots of the impedance magnitude from $10 \mathrm{~Hz}$ to $10 \mathrm{kHz}$ during the three distinct time periods yield additional insight into the developing electrical characteristics of the recording sites over time (figure 4 ). Ions can more effectively infiltrate the porous PEDOT at lower frequencies, creating a larger accessible interfacial area and therefore reducing lowfrequency impedance by increasing recording site capacitance and lowering charge transfer resistance [16]. On day 1 after the surgery, the mean impedance magnitude of the PEDOT sites at very low frequencies $(\leqslant 40 \mathrm{~Hz})$ was more than 30 times smaller than the uncoated sites (figure 4). On day 9 during the peak of the early reactive response, the mean impedance magnitude of the PEDOT sites at very low frequencies was still more than 

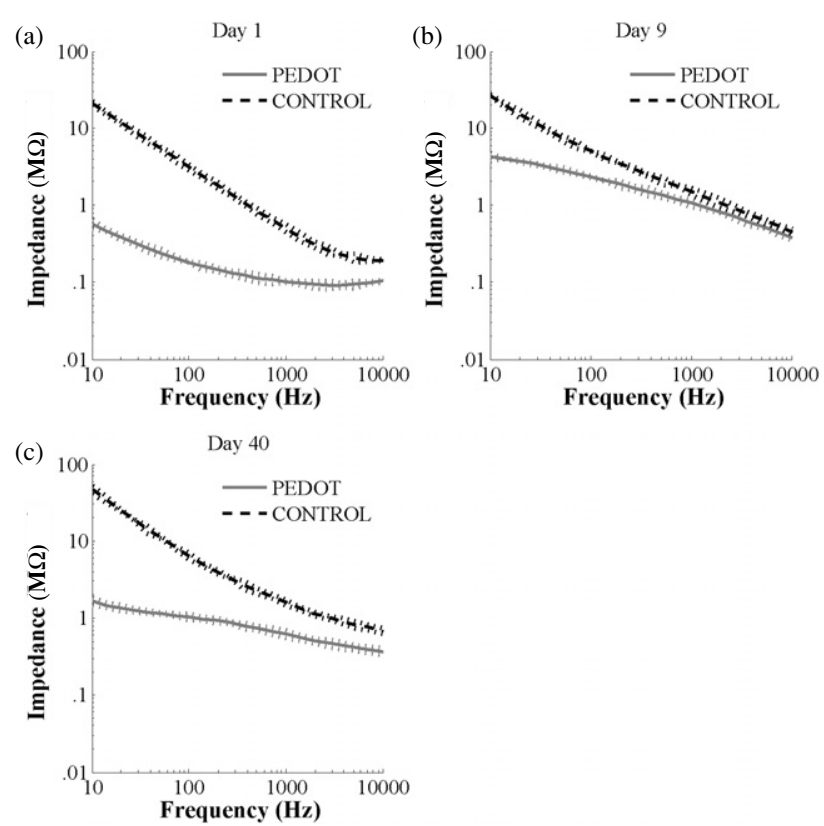

Figure 4. Bode plot of average measured impedance versus frequency. The dotted lines denote standard error of the data set on a given day $(n=64)$. (a) Day 1 post-implantation. (b) Day 9 postimplantation. (c) Day 40 post-implantation. Initially, there was a large difference in impedance between PEDOT and control sites at both $1 \mathrm{kHz}$ and low frequencies $(10-40 \mathrm{~Hz})$. On day 9, the difference in impedance at $1 \mathrm{kHz}$ was much smaller, whereas a large difference in impedance at low frequencies was still evident. By day $41,1 \mathrm{kHz}$ and low-frequency impedance for both PEDOT and control sites had settled. A large difference in impedance at low frequencies between PEDOT and control sites remained; however, the difference in impedance at $1 \mathrm{kHz}$ was not as dramatic as seen initially.

ten times smaller than the uncoated sites (figure 4). On day 40, the mean impedance magnitude of the PEDOT sites returned to almost 30 times smaller than the uncoated sites (figure 4). The trend in impedance of the PEDOT sites over time at low frequency also parallels the time course of initial trauma, early reactive response and sustained immune response.

\subsection{Unit recordings}

Over the course of the experiment, signals that regularly exceeded the 3.5 standard deviation threshold were evident on all 128 recording sites. On average, $70 \%$ of the PEDOT sites recorded waveforms categorized as poor or better (SNR $>2)$, compared to $59 \%$ for the uncoated sites $(p<0.001)$ (figure 5 ). After day $15,78 \%$ of the PEDOT sites recorded waveforms categorized as poor or better, in contrast to $68 \%$ for the control sites $(p<0.001)$. The overall percentage of sites recording poor or better unit recordings was high initially, markedly lower for the two weeks following the surgery and then returned to prior levels after the two-week mark. Multiple units were evident on a number of both PEDOT and uncoated recording sites. An average of $1.2 \pm 0.3$ units of poor or better quality were identified on PEDOT sites over the course of the experiment, while an average of $1.0 \pm 0.3$ units of poor or better quality identified on control sites $(p<0.001)$.
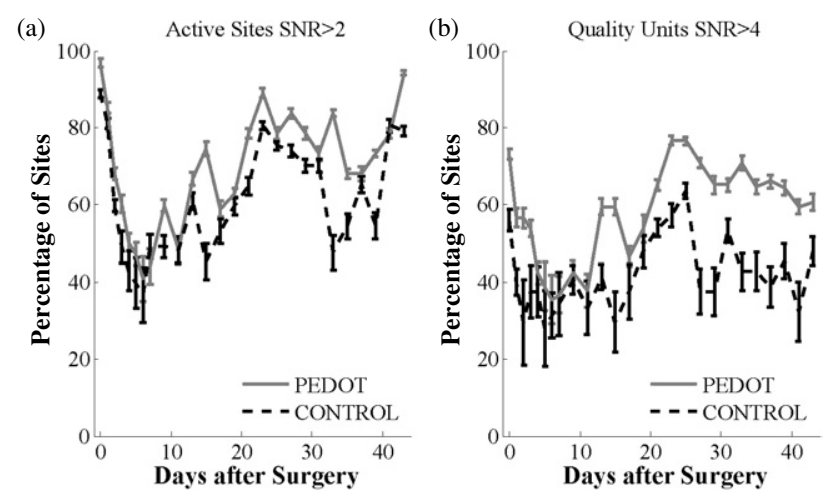

Figure 5. Percentage of sites recording low- and high-quality units on a given day. The bars denote standard error of the data set on a given day $(n=8)$. (a) Units with $S N R>2$. (b) Quality units with $\mathrm{SNR}>4$. Unit recordings tended to be unstable over the first two weeks after implantation. During these two weeks, a noticeable drop in measurable units occurred on both PEDOT and control sites. After two weeks, unit recordings stabilized and the recordable number of units became more constant on a day-to-day basis. Throughout the course of the study, sites electrochemically deposited with PEDOT films on average registered both more lowand high-quality units than control sites $(p<0.001)$.

A more dramatic difference in performance between PEDOT and uncoated sites over the length of the study is evident in the percentage of sites recording high-quality units (SNR > 4) (figure 5). Over the six-week study, an average of $41 \%$ of the uncoated recording sites at any given time registered at least 1 quality unit. In comparison, more than $58 \%$ of the PEDOT sites registered at least 1 quality unit ( $p<$ 0.001 ). PEDOT sites recorded an average of $0.75 \pm 0.1$ quality units from day to day, whereas uncoated sites recorded an average of $0.56 \pm 0.1$ quality units $(p<0.001)$.

\subsection{Signal to noise over recording sessions}

Calculating an average signal-to-noise ratio (SNR) of unit recordings was complicated by the fact that PEDOT sites registered a larger number of poor, moderate and quality units. For purposes of calculating the SNR of unit recordings, only units of moderate or better quality $(\mathrm{SNR}>3.0)$ were used. The SNR of recorded units of moderate or better quality over time can also be separated into three time periods (figure 6). During the two days following surgery, the average SNR of the units recorded on PEDOT sites was $5.1 \pm 1.2$ while the average SNR of the units recorded on the control sites was $4.1 \pm$ $1.1(p<0.001)$. Over the period spanning days three to fifteen following surgery, the average SNR of units recorded on PEDOT and uncoated sites was roughly the same, $4.8 \pm$ 1.8 and $4.9 \pm 1.7$, respectively $(p>0.05)$. After day 15 , the SNR of unit recordings for PEDOT and uncoated sites began to diverge again; the average SNR of units recorded on PEDOT sites was $5.1 \pm 1.2$, while the average SNR of units recorded on uncoated sites was $4.3 \pm 1.0(p<0.001)$. 


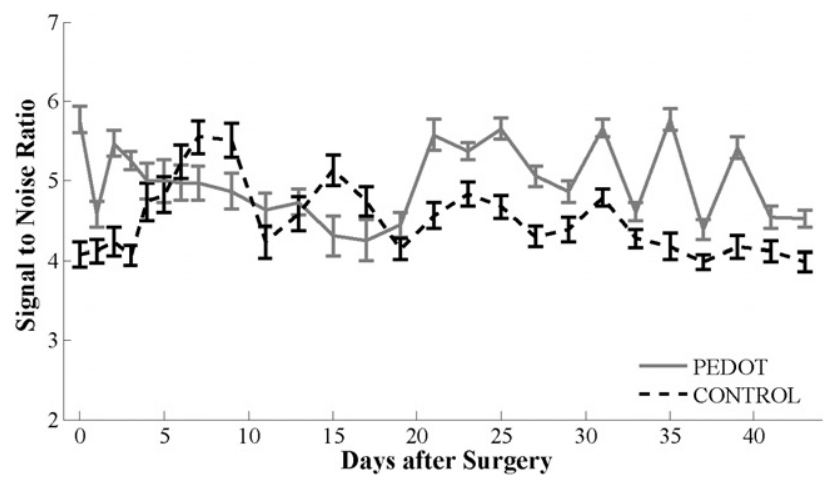

Figure 6. Average SNR over time. The bars denote standard error of the data set on a given day $(n=64)$. Average SNR was calculated using units with an SNR of greater than 3, as PEDOT sites typically registered both more low- and high-quality units. On average, PEDOT sites recorded units with greater SNR than control sites for the first three days after implantation and from day 15 until the end of the study $(p<0.001)$. Between day 3 and day 15 postimplantation, individual unit recordings varied on day-to-day (and even hour-to-hour) basis. No significant difference in average SNR between PEDOT and control sites was noted during this timeframe $(p>0.05)$.

\subsection{Local field potential recordings}

Local field potentials recordings (LFPs), typically ranging from 3 to $90 \mathrm{~Hz}$, are routinely recorded using Michigan probes. Over the course of taking chronic LFP recordings, an undesirable low-frequency artifact often becomes evident [48-50]. LFP recordings were taken at the end of the sixweek study in order to investigate the possibility that sites deposited with a PEDOT film would reduce low-frequency artifact in LFP recordings. Figure 7 depicts a sample of LFPs recorded on PEDOT and control sites. Low-frequency artifact was intermittently evident on the control recording sites that was not apparent on the PEDOT sites. The magnitude of the average power in the band from 0.1 to $1 \mathrm{~Hz}$ is indicative of the extent of low-frequency artifact. Across all subjects, the average power in the frequency band from 0.1 to $1 \mathrm{~Hz}$ for the control sites was $4.3 \pm 0.8$ times larger than the average power across the same band for PEDOT sites $(p<0.001)$. For both types of sites, the low-frequency artifact was sufficient in amplitude to register despite the use of a second-order bandpass filter with a cutoff frequency of $3 \mathrm{~Hz}$.

\subsection{Histological evaluation}

Although detailed histological evaluation was not a focus of this study, cursory hematoxylin and eosin ( $\mathrm{H}$ and $\mathrm{E})$ staining was performed on the implanted cortical tissue from six of the subjects at the end of the experiment (data not shown) [31, 51]. Since the probes were inserted by hand, the insertion angle of the probe relative to the surface of the brain could not be guaranteed. As a result, determining the precise location of a specific recording site relative to a coronal section was impossible and therefore no specific comparison between immune response at PEDOT and control sites could be made. Coronal sections were instead visually inspected for any
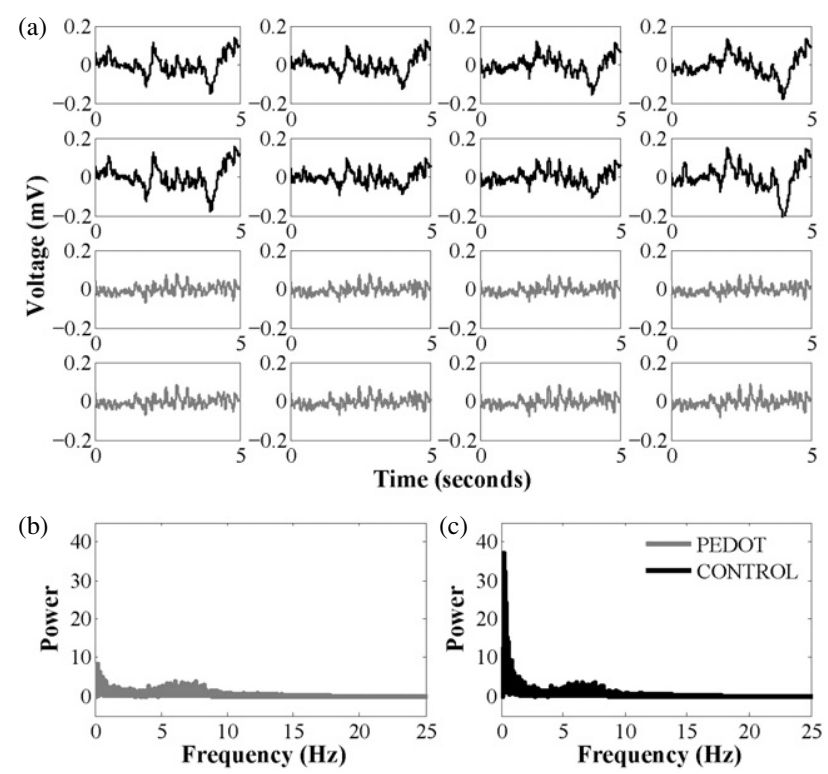

Figure 7. Local field potential recordings. (a) Five-second segment of local field potential recordings for one subject on day 41 . The top two rows are local field potential recordings from control sites. The bottom two rows are local field potential recordings from sites deposited with a PEDOT film over the same timeframe. Sites deposited with a PEDOT film registered considerably less low-frequency artifact $(0.1-1 \mathrm{~Hz})$ than control sites. (b) Average power versus frequency (resolution $=0.001 \mathrm{~Hz}$ ) of PEDOT sites for same subject on day 41. (c) Average power versus frequency (resolution $=0.001 \mathrm{~Hz}$ ) of control sites for same subject on day 41 .

abnormally large immune response. In general, the coronal sections were consistent with the modest global tissue reaction to implanted silicon probes reported elsewhere [31, 45, 52].

\section{Discussion}

\subsection{Trend in recordings with respect to progression of the immune response}

There have been a number of excellent studies monitoring the progression of the immune response to an implanted silicon probe over time [44-46, 52]. In addition, Liu and McCreery have investigated the stability of single-unit neural recordings at different points in time after implantation [53]. In order to place the comparative results of PEDOT and control recording sites into proper context, a discussion of the trends in recording site impedance, recorded signal amplitude and recorded noise amplitude over the six weeks following implantation is necessary. The trends found in this study follow logically from the known progression of the immune response to an implanted silicon probe and are consistent with results from similar microelectrode studies [31, 51, 54].

Immediately upon implanting the modified probes, the measured impedance on all recording sites increased by 70 $100 \mathrm{k} \Omega$. This impedance increase may be attributed to immediate protein adsorption on the recording sites coupled with the change in the surrounding medium from $0.1 \mathrm{M}$ phosphate buffered saline to cortical tissue. As the individual 


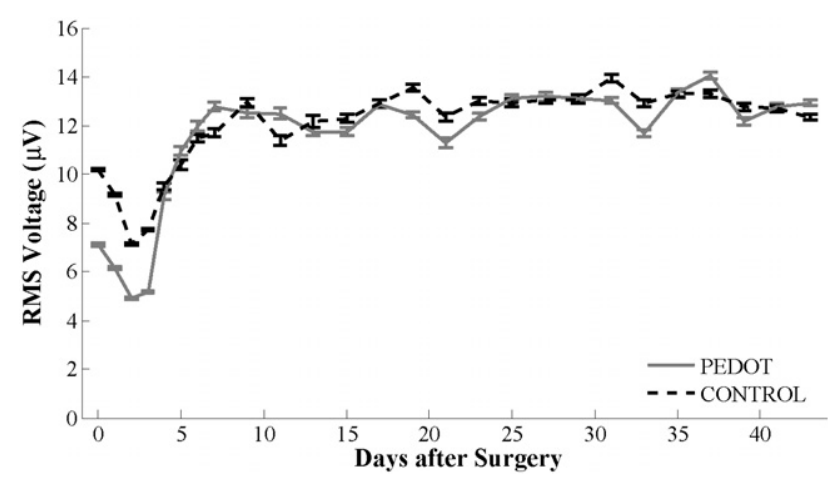

Figure 8. Average noise over time. The bars denote standard error of the data set on a given day $(n=64)$. The recorded noise level for PEDOT sites was significantly lower than control sites over the first three days following implantation, presumably a result of a reduced thermal noise $(p<0.001)$. The recorded noise level for both PEDOT and control sites increased dramatically after day 3 , reaching a maximum value at the one-week mark. The increase in impedance on both PEDOT and control sites apparent after day 3 should also cause an increase in thermal noise. By day 15 postimplantation, the average RMS noise on PEDOT and control sites was not significantly different $(p>0.05)$.

shanks of the Michigan probe are small in comparison to other recording technologies, a minimal amount of tissue should be disrupted during their insertion. As a result, recordings with the Michigan probe immediately after surgery tend to be strong $[55,56]$.

According to the literature, swelling of the tissue surrounding an implanted biomaterial occurs not long after implantation [57]. Swelling around an implanted probe could cause neurons near the recording sites to be 'pushed away'. As a result of swelling, the neural sources of signal could be moved to a more distant location, theoretically causing a decrease in signal amplitude. Similarly, the noise amplitude would decrease as the distal neural sources that generate the biological component of measured noise are also pushed away from the recording site. Consistent with this hypothesis, both measured signal amplitude as well as recorded noise amplitude in this study decreased following surgery. In general, swelling subsides between three and six days after a biomaterial is implanted [57], theoretically allowing displaced neurons to return to the area around the recording site. In this study, both signal and noise amplitude increased during days three to seven, which is consistent with neurons returning to the region near the recording site as swelling subsides (figures 8 and 9).

Biran et al report that there is a comparable reduction in neuronal cell density in the tissue surrounding an implanted probe at two and four weeks post-insertion [52]. Data tracking neuronal cell density surrounding a probe over the two weeks following implantation is not currently available; however, it may be possible that this reduction in neuronal cell density develops immediately after a probe is inserted and remains relatively constant for the life of the implant. A reduction in neuronal cell density should result in an overall decrease in measured signal and biological noise and therefore could also account for the reduction in recorded signal and noise amplitude that occurred after the first day after implantation.

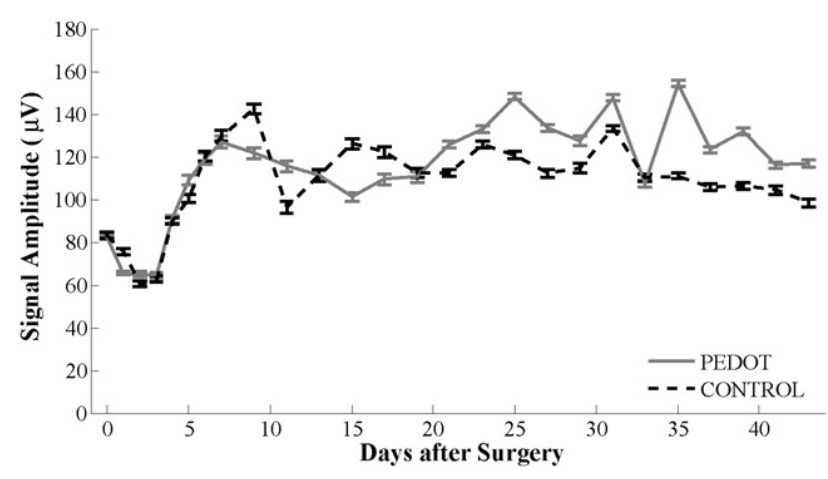

Figure 9. Average signal amplitude over time. The bars denote standard error of the data set on a given day $(n=64)$. During the first three days after implantation, swelling around the recording sites may have pushed neurons away from the recording, causing an apparent reduction in signal amplitude. Average signal amplitude was not significantly different between PEDOT and control sites during this timeframe $(p>0.05)$. Signal amplitude increased until seven days post-surgery. At the two-week point day-to-day recordings stabilized and the average signal amplitude of units recorded on PEDOT sites became significantly larger in comparison to control sites $(p<0.001)$.

The reduction in recorded signal and noise amplitude as a result of decreased neuronal cell density should be a relatively constant bias over time and therefore does not account for the dramatic increase in recorded signal and noise apparent after day 3 in this study.

Unit recordings over the two weeks following surgery were unstable. Individual unit waveforms and PCA clusters changed dramatically on a daily, and sometimes hourly, basis. Although detailed statistical analysis on the stability of unit recordings was not performed in this study, unit recordings were considered unstable if large variations in the PCA cluster for a given neuron were evident, in conjunction with a noticeable change in the shape or amplitude of the recorded waveform. Unit recordings are unstable during the early reactive response timeframe because the tissue surrounding the recording sites is in a constant state of flux [46, 53]. During this period, a loose sheath of cells consisting of microglia and reactive astrocytes begins to form around the shanks of the recording electrodes [46]. The impedance of the electrode recording sites at $1 \mathrm{kHz}$ in this study increased up to the twoweek mark (figure 3, table 1), presumably as a result of the formation of a cellular sheath.

Researchers have shown that the encapsulation around the electrode shanks becomes more defined at the two-week mark, and the immune response begins a transition into the chronic phase [46]. Around the two-week point in this study, the dayto-day and site-to-site variability in impedance magnitude at $1 \mathrm{kHz}$ diminished (figure 3) [31]. Unit recordings became more stable; individual unit recordings changed primarily on a week-to-week basis. Liu and McCreery suggest that the encapsulation of the electrode shank anchors the recording site in position with respect to the surrounding tissue and therefore stabilizes the day-to-day recordings [53].

By the sixth week after surgery, a well-defined encapsulating sheath of microglia and reactive astrocytes has 
formed around typical implanted silicon devices, and the immune response has transitioned into the long-term chronic response [46]. Presumably, as a result of this encapsulating sheath, the $1 \mathrm{kHz}$ impedances at six weeks for all recording sites in this study were between 500 and $1000 \mathrm{k} \Omega$ larger than initial in vitro impedance measurements (figure 3) [31]. Consistent with the findings of Liu and McCreery, unit recordings at six weeks became even more stable [53].

\subsection{Effect of reduced impedance on recorded signal and noise amplitude}

Lowering the impedance of recording sites is purported to enhance neural recordings by reducing noise and minimizing signal loss through shunt pathways [7, 8, 11, 12]. Noise in neural recordings is primarily comprised of thermal (Johnson) noise and small amplitude signals from diffuse neural sources $[7,8,11]$. In vitro, the largest component of recorded noise is thermal noise. The thermal noise for a recording site can be computed as follows:

$$
V_{\text {Noise }}=\sqrt{4 k T Z \Delta F}
$$

where $k$ is Boltzman's constant, $T$ is the temperature, $\Delta F$ is the frequency band of interest and $Z$ is the impedance at the frequency band of interest [7, 8, 11]. As long as the thermal noise remains the largest portion of the measured noise, the recorded noise should vary proportionately with the measured impedance over the frequency band of interest. The average in vitro noise amplitude for PEDOT recording sites was $2.1 \pm$ $0.3 \mu \mathrm{V}$, compared to $5.5 \pm 0.3 \mu \mathrm{V}$ for control sites $(p<0.001)$. A reduction in the impedance of an electrode site should not only decrease thermal noise, but increase both measured signal as well as measured biological noise, due to a reduction in measured potential loss through shunt pathways.

The ratio of impedance at $1 \mathrm{kHz}$ between uncoated sites and PEDOT sites was approximately 7.5 to 1 over the first two days following surgery. Consequently, the average measured noise was $8.0 \pm 0.4 \mu \mathrm{V}$ for uncoated sites and $5.3 \pm 0.3 \mu \mathrm{V}$ for PEDOT sites $(p<0.001)$ (figure 8). This increase in measured noise amplitude for PEDOT and control sites with respect to in vitro values is attributable to a $70 \mathrm{k} \Omega$ increase in impedance after implantation across all sites coupled with the addition of biological sources of noise. Over the same timeframe, the average signal amplitude for units recorded on PEDOT sites was not significantly different from the average signal amplitude of units recorded on control sites $(p>0.05)$ (figure 9). Therefore, the difference in SNR between PEDOT and uncoated sites over the first two days was attributable to diminished thermal noise (figure 6). Swelling around the recording site during this timeframe likely increased the distance from the recording site to signal sources and sources of biological noise, mitigating the benefit of decreased signal loss through the shunt pathway. The increased SNR attributable to decreased thermal noise related directly to an increase in the resolvable number of neurons (figure 5).

From day 3 to day 15 , the impedance magnitude at $1 \mathrm{kHz}$ increased on both uncoated and PEDOT sites, presumably as a result of the early reactive response to the implants. Similarly, the average noise voltage for uncoated and PEDOT sites increased to $11.9 \pm 0.6 \mu \mathrm{V}$ and $11.4 \pm 0.5 \mu \mathrm{V}$, respectively $(p>0.05)$. As the ratio of impedance at $1 \mathrm{kHz}$ between uncoated and PEDOT sites diminished, the average noise amplitude for both types of sites converged. The average signal amplitude for units recorded between day 3 and day 15 increased to $112 \pm 15 \mu \mathrm{V}$ for PEDOT sites and to $115 \pm 17 \mu \mathrm{V}$ for control sites $(p>0.05)$.

As discussed earlier, the dramatic increase in both measured noise and measured signal amplitude was likely related to the return of neurons to the area surrounding the recording site after the insertion trauma subsided. As neurons returned, the contribution of the diffuse activity of neurons nearby the recording sites to the ambient noise level began to approach and even surpass the contribution of thermal noise. Moreover, the thermal noise presumably increased in proportion to the increase in impedance over all sites. Because neither the average signal amplitude nor recorded noise for PEDOT and uncoated sites was markedly different between days 3 and 15, the SNR for both PEDOT and control sites was approximately the same. Although more units were evident on PEDOT sites during this period (figure 5), the unit recordings were very unstable on a day-to-day (and sometimes hourto-hour) basis during this timeframe, complicating statistical analysis.

After day 15, the impedance magnitude at $1 \mathrm{kHz}$ settled for both PEDOT and uncoated sites. Action potentials recorded from individual neurons became consistent on a week-to-week basis. The difference in average noise amplitude between PEDOT and uncoated sites remained negligible (PEDOT: $12.7 \pm 0.8 \mu \mathrm{V}$, uncoated: $13.1 \pm 0.9 \mu \mathrm{V}, p>0.05)$. However, the average signal amplitude of units recorded on PEDOT sites increased to $129 \pm 15 \mu \mathrm{V}$ after day 15 , while the average signal amplitude of units recorded on uncoated sites did not change significantly $(113 \pm 13 \mu \mathrm{V}, p<0.001)$. Consequently, the SNR of units recorded on PEDOT sites after day 15 was higher than the SNR of units recorded on uncoated sites, correlating directly to an increased number of sorted units (figure 5).

The increase in signal amplitude on PEDOT sites after day 15 is most likely a function of decreased signal loss through the shunt impedance ${ }^{7}$. Similarly, a decrease in signal loss through the shunt impedance should increase the amplitude of measured biological noise. As a result, the noise amplitude across PEDOT sites approached the noise amplitude across control sites; the expected decrease in thermal noise caused by reduced impedance was compensated for by an increase in measured biological noise.

\subsection{Functional significance of reduced initial impedance on single-unit recordings}

Prior to implantation and over the first three days following surgery, every PEDOT site in this study registered a lower

7 Additionally, conductive polymer coatings grow outward from the normally planar recording site to create a hemispherical electrode site, enhancing the site's ability to detect neurons in the periphery [16]. The rough topography of a conductive polymer film has also been hypothesized to promote neural growth in comparison to planar sites [16]. Over multiple animals, these effects may create an additional increase in both average signal amplitude as well as the average measured amplitude of biological noise sources. 
measured noise amplitude than the lowest measured noise amplitude for a control site. This result is likely attributable to the large difference in $1 \mathrm{kHz}$ impedance seen between PEDOT and control sites during this period. After day 3, there was an equivalent increase in impedance on both PEDOT and control sites, indicating a global immune response affecting the electrical characteristics of PEDOT and control sites equally. As site impedances increased by $0.5-1 \mathrm{M} \Omega$ with the encapsulation of the probe, the ratio of average impedance at $1 \mathrm{kHz}$ between control and PEDOT sites decreased from the 10 to 1 in vitro value to 2 to 1 . Consequently, the benefit of electrochemically depositing PEDOT films in order to improve single-unit recordings was mitigated. This result is not surprising considering that the expected difference in thermal noise and signal loss through shunt impedance between PEDOT and control sites depends on the magnitude of this ratio.

Although a statistically significant increase in signal to noise and number of recorded units was evident using a large number of recording sites over an extended period of time, the improvement in recordings for individual subjects on specific days was not always significant. Alternative methods intended to reduce the initial impedance of neural recording electrodes will also likely have their efficacy diminished by the contribution of encapsulation to recording site impedance. Reducing initial impedance is of some benefit, but these results suggest that the single largest determinant in effectively isolating a single-unit potential in a chronic setting is distance from recording site to neuronal source.

There is a possibility that the PEDOT coatings delaminated partially in vivo, which may also cause an increase in recording site impedance. Attempts were made to explant the intact recording electrodes from the animals at the end of study to examine the possibility of delamination, but none of these attempts were successful. Unfortunately, the process of explanting chronic recording electrodes (which includes dissolving the acrylic headcap with 1,2-dichloroethane solution, removing the surrounding skull from the brain and physically extracting a probe from the surrounding tissue that has been encapsulated with astrocytes and microglia) causes considerable strain on both the electrode shanks and fragile silicon connecting cable. As a result, the process of explantation can cause delamination of PEDOT coatings and therefore is problematic in evaluating the stability of PEDOT coatings in vivo.

The data in this study suggest that delamination of the PEDOT films was not a significant factor in the results. Statistically significant differences in impedance between PEDOT and control sites were observed across all days of the study, including a dramatic difference in impedance at low frequency. Moreover, an equivalent impedance increase at $1 \mathrm{kHz}$ was observed for both PEDOT and control sites, which suggests that the source of the impedance increases in this study was acting globally, instead of site-specific delamination. Finally, the time course and magnitude of the impedance increases observed across all sites were consistent with the immune response to implanted recording devices seen in other studies [31, 46, 51].

\subsection{Artifact in LFP recordings}

Low-frequency noise in LFP recordings is often the result of either motion artifact or thermal noise. As noted earlier, thermal noise is proportional to the square root of impedance across a frequency band. Standard iridium electrodes are designed to transduce ionic current into electronic current via the formation of a non-faradaic capacitive double layer. The impedance of this capacitive double layer at a given frequency is $1 / \mathrm{j} \omega C$, where $\omega$ is the frequency and $C$ is the double layer capacitance. LFP recordings typically have frequency components in the $3-90 \mathrm{~Hz}$ range, much lower than the $350-5000 \mathrm{~Hz}$ range of action potentials. Not surprisingly, the impedances of iridium microelectrodes at frequencies relevant for LFP recordings are substantially higher than their impedances at $1 \mathrm{kHz}$ (figure 4).

Recording sites deposited with PEDOT films in this study routinely exhibited much lower impedances than iridium control sites at low frequencies (figure 4 , section 3 ). As noted earlier, ions can more effectively infiltrate the porous PEDOT at lower frequencies, creating a larger accessible interfacial area and therefore reducing low-frequency impedance by increasing recording site capacitance and lowering charge transfer resistance [16]. At the six-week mark, the ratio in average impedance at low frequency between control and PEDOT sites was still quite large (section 3). A large decrease in low-frequency impedance should correlate directly to a reduction in thermal noise across lower frequency bands. However, the relative magnitude of thermal noise in proportion to the magnitude of the desired LFP signal of interest is unknown. Furthermore, thermal noise should be random in nature, whereas the low-frequency artifact visually evident across control sites was highly correlated (Spearman's rank coefficient test $p<0.001$ ) (figure 7).

Artifact that is highly correlated across multiple sites suggests one outside source of artifact influencing the recordings on all of those sites, such as motion artifact. Motion artifact is often the result of a disturbance in the established capacitive double layer at the interface between the recording site and the tissue. One possible source of motion artifact is movement of the electrode shanks with respect to the surrounding tissue [58]. Movement of the electrode shanks can cause a disturbance in the ionic layer at multiple recordings sites simultaneously. Because the impedance of iridium electrode sites at low frequencies is quite high, disturbing the ionic interface could result in large variations in measured potential. PEDOT-coated recording sites, having considerably reduced low-frequency impedance, would therefore register smaller variations in measured potential when the ionic interface is disturbed.

An alternative possible explanation for the apparent resistance of PEDOT films to low-frequency artifact relates to the mechanism by which PEDOT films transduce ionic current into electronic current. Unlike non-faradaic noble metal microelectrodes, PEDOT films are designed to transduce ionic current into electronic current via a reversible faradaic redox reaction. The increased impedance of the non-faradaic capacitive double layer at low frequencies may cause a bias toward a faradaic mechanism of conduction for PEDOT films. 
Faradaic electrode reactions are governed by the concentration of product and reactants species and the mobility of electrons and ions in their respective mediums. Porous PEDOT films are designed to promote fast ion exchange at the interface between recording site and surrounding tissue $[15,17]$. As a result, motion of the electrode with respect to the surrounding tissue may not significantly disturb the local concentration of product and reactants. Consequently, LFPs taken with PEDOT sites may be more resistant to motion artifact.

Reducing the amount of low-frequency artifact in LFP recordings has a number of potential benefits. First, removing artifact closer to the source is always preferential to digital filtering. Second, digital filtering of the low-frequency artifact may not be an option for real-time applications. Third, large low-frequency artifact can cause the recording amplifier to saturate, preventing any viable signal from being registered. Finally, recording electrode low-frequency drift is often a limiting consideration in device design.

\section{Conclusion}

Over the course of the study, recording sites deposited with a PEDOT film registered $17 \%$ more quality units than control sites, while minimizing the amount of low-frequency artifact evident in LFP recordings. The modest tissue response, typical of cortical implants, resulted in an average $700 \mathrm{k} \Omega$ increase in impedance magnitude at $1 \mathrm{kHz}$ across both PEDOT and control recording sites at six weeks. Consequently, the benefit of reduced noise and decreased signal loss through the shunt impedance as a function of lower initial impedance for PEDOT films was diminished. The dominating contribution to site impedance of the encapsulating sheath should similarly affect alternative methods of reducing initial recording site impedance. The results of this study indicate that surfactanttemplated ordered PEDOT films remain suitable for obtaining high-quality neural recordings out to six weeks. Future studies will investigate the long-term effectiveness of seeding PEDOT films with drugs tailored to alleviate the immune response and induce neural growth toward the electrode in order to maximize recording performance.

\section{Acknowledgments}

The authors of this paper would like to acknowledge all of members of the Neural Engineering Laboratory at the University of Michigan for their assistance in this study. Specific thanks go to Elizabeth Nunamaker and Erin Purcell for histological assistance, Rachel Miriani and Jey Subbaroyan for surgical assistance and Rio Vetter, Matt Johnson and Nick Langhals for invaluable advice throughout the course of the study. David C Martin acknowledges partial support provided by NIH NINDS-N01-NS-1-2338 and NSF DMR 0084304 , NSF DMR 0518079.

\section{References}

[1] Abbott L F and Salinas E 1994 Vector reconstruction from firing rates J. Comput. Neurosci. $189-107$
[2] Beiko J and Cain D P 1998 The effect of water maze spatial training on posterior parietal cortex transcallosal evoked field potentials in the rat Cereb. Cortex 8 407-14

[3] Brown E N, Frank L M, Tang D, Quirk M C and Wilson M A 1998 A statistical paradigm for neural spike train decoding applied to position prediction from ensemble firing patterns of rat hippocampal place cells J. Neurosci. 18 7411-25

[4] Carmena J M et al 2003 Learning to control a brain-machine interface for reaching and grasping by primates $P L O S$ Biol. 1 e 42

[5] Dobelle W H 2000 Artificial vision for the blind by connecting a television camera to the visual cortex ASAIO J. 46 3-9

[6] Serruya M D, Hatsopoulos N G, Paninski L, Fellows M R and Donoghue J P 2002 Instant neural control of a movement signal Nature 416 141-2

[7] Kovacs G T A 1994 Introduction to the theory, design, and modeling of thin-film microelectrodes for neural interfaces Enabling Technologies for Cultured Neural Networks ed D A Stenger and T McKenna (New York: Academic) pp 121-65

[8] Schmidt E and Humphrey D R 1990 Extracellular single-unit recording methods Neurophysiol. Tech. (Clifton, NJ: Humana Press) pp 1-64

[9] Paik S J, Park Y and Cho D I 2003 Roughened polysilicon for low impedance microelectrodes in neural probes J. Micromech. Microeng. 13 373-9

[10] Drake K L, Wise K D, Farraye J, Anderson D J and BeMent S L 1988 Performance of planar multisite microprobes in recording extracellular single-unit intracortical activity IEEE Trans. Biomed. Eng. 35 719-32

[11] Shoham S and Nagarajan S 2003 The theory of central nervous system recording Neuroprosthetics: Theory and Practice ed KW Horch and G S Dhillon (Singapore: World Scientific) pp 448-65

[12] Robinson D A 1968 The electrical properties of metal microelectrodes Proc. IEEE 561065

[13] Burke L D and Shannell R A 1984 An investigation of hyrdous oxide growth on iridium in base $J$. Electroanal. Chem. 119-41

[14] Burke L, Mulcahy J K and Whelan D P 1984 Prepartion of an oxidized iridium electrode and the variation of its potential with pH J. Electroanal. Chem. 117-28

[15] Cui X and Martin D C 2003 Electrochemical deposition and characterization of poly(3,4-ethylenedioxythiophene) on neural microelectrode arrays Sensors Actuators B 89 92-102

[16] Cui X Y, Hetke J F, Wiler J A, Anderson D J and Martin D C 2001 Electrochemical deposition and characterization of conducting polymer polypyrrole/PSS on multichannel neural probes Sensors Actuators A 93 8-18

17] Yang J, Kim D H, Hendricks J L, Leach M, Northey R and Martin D C 2005 Ordered surfactant-templated poly(3,4-ethylenedioxythiophene) (PEDOT) conducting polymer on microfabricated neural probes Acta Biomater. 1 125-36

[18] Cui X Y et al 2001 Surface modification of neural recording electrodes with conducting polymer/biomolecule blends J. Biomed. Mater. Res. 56 261-72

[19] Bobacka J, Lewenstam A and Ivaska A 2000 Electrochemical impedance spectroscopy of oxidized poly (3,4-ethylenedioxythiophene) film electrodes in aqueous solutions J. Electroanal. Chem. 489 17-27

[20] Craighead H G, Hames C D and Turner A M P 2001 Chemical and topographic patterning for directed cell attachment Curr. Opin. Solid State Mater. Sci. 177-84

[21] Dowell-Mesfin N M et al 2004 Topographically modified surfaces affect orientation and growth of hippocampal neurons J. Neural Eng. 1 78-90 
[22] Fan Y W C F Z, Chen L N, Zhai Y, Xu Q Y and Lee I S 2002 Adhesion of neural cells on silicon wafer with nano-topographical surface Appl. Surf. Sci. 187 313-8

[23] St John P M et al 1997 Preferential glial cell attachment to microcontact printed surfaces $J$. Neurosci. Methods 75 171-7

[24] Schmidt C E, Shastri V R, Vacanti J P and Langer R 1997 Stimulation of neurite outgrowth using an electrically conducting polymer Proc. Natl Acad. Sci. USA 94 8948-53

[25] Beck F, Braun P and Oberst M 1987 Organic electrochemistry in the solid state-overoxidation of polypyrrole $\mathrm{Ber}$. Bunsenges. Phys. Chem. Chem. Phys. 91 967-74

[26] Hitoshi Yamato M O and W Wernet 1995 Stability of polypyrrole and poly(3,4-ethylenedioxythiphene) for biosensor application J. Electroanal. Chem. 397 163-70

[27] Schlenoff J B and Xu H 1992 Evolution of physical and electrochemical properties of polypyrrole during extended oxidation J. Electrochem. Soc. 139 2397-401

[28] Wernet W 1985 PhD Thesis Freidburg

[29] Anderson D J et al 1989 Batch-fabricated thin-film electrodes for stimulation of the central auditory system IEEE Trans. Biomed. Eng. 36 693-704

[30] Shahriari K 2001 Safe and effective techniques for surgically inserting flexible microelectrode arrays into the cortex Thesis M S Arizona State University

[31] Vetter R J, Williams J C, Hetke J F, Nunamaker E A and Kipke D R 2004 Spike recording performance of implanted chronic silicon-substrate microelectrode arrays in cerebral cortex IEEE Trans. Neural Syst. Rehabil. Eng. 52 896-904

[32] Gerstein G L, Bloom M J, Espinosa I E, Evanczuk S and Turner M R 1983 Design of a laboratory for multineuron studies IEEE Trans. Syst. Man Cybern. 13 668-76

[33] Glaser E M 1971 Separation of Neuronal Activity by Waveform Analysis (New York: Academic)

[34] Glaser E M and Marks W B 1968 On-line separation of interleaved neuronal pulse sequences Data Acquis. Process. Biol. Med. 5 137-56

[35] Lewicki M S 1998 A review of methods for spike sorting: the detection and classification of neural action potentials Netw. Comput. Neural Syst. 9 R53-78

[36] Dunn J C 1974 A fuzzy relative of the ISODATA process and its use in detecting compact well compact well-separated clusters J. Cybern. 3 32-57

[37] Bezdek J C 1981 Pattern Recognition with Fuzzy Objective Function Algorithms. (New York: Plenum)

[38] Zouridakis G and Tam D C 2000 Identification of reliable spike templates in multi-unit extracellular recordings using fuzzy clustering Comput. Methods Programs Biomed. 61 91-8

[39] Karkkainen I and Franti P 2002 Dynamic local search for clustering with unknown number of clusters Int. Conf. on Pattern Recognition (August 2002, Quebec, Canada) p 240-3

[40] Henze D A, Borhegyi Z, Csicsvari J, Mamiya A, Harris K D and Buzsaki G 2000 Intracellular features predicted by extracellular recordings in the hippocampus in vivo J. Neurophysiol. 84 390-400

[41] Suner S, Fellows M R, Vargas-Irwin C, Nakata G K and Donoghue J P 2005 Reliability of signals from a chronically implanted, silicon-based electrode array in non-human primate primary motor cortex IEEE Trans. Neural. Syst. Rehabil. Eng. 13 524-41

[42] Harris K D, Henze D A, Csicsvari J, Hirase H and Buzsaki G 2000 Accuracy of tetrode spike separation as determined by simultaneous intracellular and extracellular measurements J. Neurophysiol. 84 401-14

[43] Johnson M D, Otto K J and Kipke D R 2005 Repeated voltage biasing improves unit recordings by reducing resistive tissue impedances IEEE Trans. Neural Syst. Rehabil. Eng. 13 160-5

[44] Schmidt S, Horch K and Normann R 1993 Biocompatibility of silicon-based electrode arrays implanted in feline cortical tissue J. Biomed. Mater. Res. 27 1393-9

[45] Szarowski D H et al 2003 Brain responses to micro-machined silicon devices Brain Res. 983 23-35

[46] Turner J N et al 1999 Cerebral astrocyte response to micromachined silicon implants Exp. Neurol. $15633-49$

[47] Kuffler S W 1976 A Cellular Approach to the Function of the Nervous System. (Sunderland, MA: Sinauer Associates)

[48] Brown P, Kupsch A, Magill P J, Sharott A, Harnack D and Meissner W 2002 Oscillatory local field potentials recorded from the subthalamic nucleus of the alert rat Exp. Neurol. 177 581-5

[49] Gail A, Brinksmeyer H J and Eckhorn R 2004 Perception-related modulations of local field potential power and coherence in primary visual cortex of awake monkey during binocular rivalry Cereb. Cortex 14 300-13

[50] Magill P J, Sharott A, Bevan M D, Brown P and Bolam J P 2004 Synchronous unit activity and local field potentials evoked in the subthalamic nucleus by cortical stimulation J. Neurophysiol. 92 700-14

[51] Williams J C 2001 Performance of chronic neural implants: measurement, modeling and intervention strategies $P h D$ Thesis Arizona State University

[52] Biran R, Martin D C and Tresco P A 2005 Neuronal cell loss accompanies the brain tissue response to chronically implanted silicon microelectrode arrays Exp. Neurol. $195115-26$

[53] Liu X, McCreery D B, Carter R R, Bullara L A, Yuen T G and Agnew W F 1999 Stability of the interface between neural tissue and chronically implanted intracortical microelectrodes IEEE Trans. Rehabil. Eng. 7 315-26

[54] Williams J, Rennaker R and Kipke D 1999 Long-term neural recording characteristics of wire microelectrode arrays implanted in cerebral cortex Brain Res. Brain Res. Protoc. 4 303-13

[55] Polikov V S, Tresco P A and Reichert W M 2005 Response of brain tissue to chronically implanted neural electrodes J. Neurosci. Methods 148 1-18

[56] Schwartz A B 2004 Cortical neural prosthetics Annu. Rev. Neurosci. 27 487-507

[57] Black J 1999 Biological performance of materials Fundamentals of Biocompatibility 3rd edn (revised and expanded) (New York: Dekker)

[58] Subbaroyan J, Martin D C and Kipke D R 2005 A finiteelement model of the mechanical effects of implantable microelectrodes in the cerebral cortex J. Neural Eng. 2 103-13 